\title{
Clinical effectiveness of palifermin in prevention and treatment of oral mucositis in children with acute lymphoblastic leukaemia: a case-control study
}

\author{
Dorina Lauritano $^{1}$, Massimo Petruzzi ${ }^{2}$, Dario Di Stasio ${ }^{3}$ and Alberta Lucchese ${ }^{3}$ \\ The aim of this study was to evaluate the efficacy of palifermin, an N-terminal truncated version of endogenous keratinocyte growth \\ factor, in the control of oral mucositis during antiblastic therapy. Twenty patients undergoing allogeneic stem-cell transplantation for \\ acute lymphoblastic leukaemia were treated with palifermin, and compared to a control group with the same number of subjects and \\ similar inclusion criteria. Statistical analysis were performed to compare the outcomes in the treatment vs. control groups. In the \\ treatment group, we found a statistically significant reduction in the duration of parenteral nutrition $(P=0.002)$, duration of mucositis \\ $(P=0.003)$ and the average grade of mucositis $(P=0.03)$. The statistical analysis showed that the drug was able to decrease the severity \\ of mucositis. These data, although preliminary, suggest that palifermin could be a valid therapeutic adjuvant to improve the quality of \\ life of patients suffering from leukaemia.
}

International Journal of Oral Science (2013) 6, 27-30; doi:10.1038/ijos.2013.93; published 20 December 2013

Keywords: acute lymphoblastic leukaemia; oral mucositis; palifermin

\section{INTRODUCTION}

Treatment of acute lymphoblastic leukaemia may present several side effects. ${ }^{1-3}$ During the conditioning regimen for haematopoietic stem cell transplantation (which includes total body irradiation or highdose chemotherapy), and immediately after the transplant, patients may present a variety of symptoms, one of the most frequent and debilitating $^{4-6}$ of which is oral mucositis. The armamentarium for the management of oral mucositis consists of prophylactic and therapeutic measures, including topically and systemically applied nonpharmacological agents, as well as pharmacotherapeutics. ${ }^{7-11}$

Up to $80 \%$ of paediatric patients with haematological malignancies undergoing chemotherapy experience some degree of mucositis. Moreover, it appears that the prevalence of mucositis in paediatric patients is even greater than that in adults, most likely due to the more rapid cell division in this patient population. ${ }^{12}$

Endogenous keratinocyte growth factor (KGF) is a $28-\mathrm{kD}$ heparinbinding member of the family of fibroblast growth factors (FGF-7) that was originally isolated from pulmonary fibroblasts as a protein with keratinocyte-stimulating activity. ${ }^{13}$ KGF stimulates the growth of epithelial cells in an extended variety of tissues, but with no direct effect on other cell types. The specificity of KGF is due to the restricted expression of KGF receptor. KGF is produced by mesenchymal cells located adjacent to the epithelium of several organs such as the epidermis, oral and lower gastrointestinal epithelium, pancreas, liver, lung, urothelium, prostate epithelium and other tissues. KGF is produced by dermal fibroblasts within the skin and by lamina propria cells of the intestines. ${ }^{14}$
Epithelial cells express KGF receptor in many tissues including the epidermis, pancreas, liver, lung and urothelium. ${ }^{15}$

In this study, we evaluate the safety and efficacy of palifermin (Kepivance), a recombinant $\mathrm{N}$-terminal truncated version of endogenous KGF with biological activity similar to that of the native protein, but with increased stability. Palifermin binds the KGF receptor, stimulating cell growth, proliferation, differentiation, and upregulation of cytoprotective mechanisms. Thus, palifermin may prevent the onset of epithelial cell apoptosis and prevent damage to the epithelial DNA, reduce the number of pro-inflammatory cytokines and increase protective enzymes against free radicals. ${ }^{16}$

Palifermin has been acknowledged as a drug that is able to decrease the incidence and duration of mucositis in patients with blood cancer who receive myelotoxic chemotherapy before undergoing bone marrow stem cell transplantation. ${ }^{15-17}$

Few studies have been performed on the efficacy of palifermin performed in paediatric patients. ${ }^{18-19}$ In this context, the aim of this work is to report new data from a paediatric cohort. We present an interventional, case-control study in which palifermin is administered only to the study group, and its effects are compared with a control group that has not been administered the drug. ${ }^{20-23}$

\section{MATERIALS AND METHODS}

From April 2010 to April 2012, we analysed data from 20 patients treated with palifermin and who were enrolled in the conditioning regimen for an allogeneic transplant of blood-borne stem cells. The

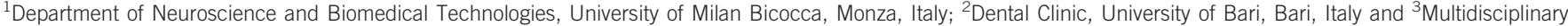
Department of Medical and Dental Specialties, Second University of Naples, Naples, Italy 
treatment group was compared to a control group of 20 subjects, with the same inclusion criteria, but who were treated with Benzydamine hydrochloride. ${ }^{24-27}$

Inclusion criteria:

- age less than 16 years;

- diagnosis of acute lymphoblastic leukaemia;

- potential allogeneic haematopoietic stem cell transplant recipient;

- scheduled to receive a myeloablative preparative regimen (cyclophosphamide/total body irradiation-based) prior to the infusion of the allogeneic graft;

- cardiac shortening fraction greater than or equal to $25 \%$;

- serum creatinine less than twice the upper limit of normal for age;

- bilirubin less than $3.0 \mathrm{mg} \cdot \mathrm{dL}^{-1}$;

- aspartate transaminase less than $500 \mathrm{IU} \cdot \mathrm{mL}^{-1}$;

- alanine transaminase less than $500 \mathrm{IU} \cdot \mathrm{mL}^{-1}$;

- amylase less than 1.5 times the upper limit of normal for age;

- lipase less than 1.5 times the upper limit of normal for age;

- no known hypersensitivity to Escherichia coli-derived proteins or palifermin;

- no active or recent (within 30 days prior to enrolment) gastrointestinal bleeding;

- no active or recent (within 30 days prior to enrolment) oral ulcerations;

- no active fungal infection, bacteraemia or viremia within 2 weeks prior to enrolment.

\section{Exclusion criteria:}

- pregnant or lactating (negative serum or urine pregnancy test within 14 days prior to enrolment).

All patients received radiotherapy in a dose of 12 Gy over eight sittings of $150 \mathrm{cGy}$ twice a day for 4 days. The children involved in the study were between the ages of 7 and 16 years.

Palifermin was administered in a dosage of $60 \mathrm{mg} \cdot \mathrm{kg}^{-1} \cdot \mathrm{d}^{-1}$ as an intravenous bolus injection for three consecutive days before and three consecutive days after myeloablative therapy (for a total of six doses).

Each patient was evaluated daily for 30 days by the same oral pathologist. Every patient with mucositis was classified, in accordance with the grading proposed by the World Health Organization (WHO) ${ }^{28}$

\section{Statistical analysis}

We divided patients in three groups (I, II, III) based on the WHO's grading for oral mucositis. Group I included grades 1 to 4; group II includes grades 2, 3 and 4; and group III included grades 3 and 4 .

For the analysis of the qualitative data, a $Z$-test ${ }^{29}$ was used to compare the incidence of $a \geqslant 1, \geqslant 2$ and $\geqslant 3$ grade of mucositis between the study group and the control group. The manifestations of mucositis were classified into groups $(\geqslant 1, \geqslant 2, \geqslant 3)$ without considering the various grading one by one. If a patient does not fall within group I, it indicates that the patient does not have any form of mucositis. The lower the frequency of group I, the greater the number of healthy patients (from the point of view of the oral affections) enrolled in the study. Accordingly, we studied the variation in the incidence of oral mucositis within the individual groups, and not the real effectiveness of Palifermin in the reduction of the grade of mucositis.

When the comparison was made between averages, and then between quantitative and not qualitative data, the Student's $t$-test. ${ }^{29}$
In both the tests, the value of statistical significance was placed at $P \leqslant 0.05$.

\section{RESULTS}

In this study, patient characteristics such as age, gender, disease and disease status at the time of haematopoietic stem cell transplantation, donor status, stem-cell dose and radiotherapy dose were comparable between the two groups. Clinical data are illustrated in Table 1. An informed consent was obtained from the parents and/or guardians of all the patients for the purposes of accurate information and to ensure maximum collaboration.

Oral mucositis of grade $\geqslant 2$ (group II) was observed in $60 \%$ of participants (12 patients) in the group that was administered palifermin and in $86 \%$ of subjects in the control group (17 patients) $(P=0.032) \quad$ (Table 2). Mucositis of grade $\geqslant 3$ (group III) was observed in of $25 \%$ of participants (5 patients) in the study group, and in 55\% (11 patients) in the control group $(P=0.154)$, while mucositis of grade $\geqslant 1$ (hence, the manifestations of mucositis in totality of their clinical manifestations) had an incidence of $75 \%$ (15 patients) in the study group and 90\% (18 patients) in the control group $(P=0.084)$ (Table 2$)$.

The average duration of the episodes of oral mucositis was 6 days when palifermin was administered (study group) and 12 days in the control group $(P=0.003)$, as shown in Table 3 . In addition, a significant difference was found in the average severity of mucositis, with a mean grading (according to the WHO classification) of 1.73 in treatment group and 2.47 in the control group $(P=0.03)$ (Table 2). Moreover, thanks to a reduction in mucositis severity, the duration of parenteral nutrition was significantly reduced as well: 15 days in the treatment vs. 16 days in the control group $(P=0.002)$ (Table 2). There was no difference in the number of documented opportunistic infections $^{30-32}$ (43\% treatment group vs. $40 \%$ in the control group) and the

Table 1 Characteristics of patients included in the study group and the control group

\begin{tabular}{lcc}
\hline Characteristics & Study group $(n=20)$ & Control group $(n=20)$ \\
\hline Age/years & $7-16$ & $7-16$ \\
Range & 11 & 11 \\
Median & 9 & 10 \\
Gender & 11 & 10 \\
Female & & \\
Male & 15 & 16 \\
Post-transplant status of disease & 2 & 1 \\
Complete remission & 3 & 4 \\
Partial remission & 6.04 & 5.85 \\
Chronic phase of disease & & \\
Dose of stem cells $\left(\times 10^{6}\right.$ per kg of & & \\
CD34*) & & \\
\hline
\end{tabular}

Table 2 Comparison between results obtained in the study group and the control group

\begin{tabular}{lccc}
\hline Results & Study group & Control group & $P$ value \\
\hline Incidence of mucositis group I & $15(75 \%)$ & $18(90 \%)$ & 0.084 \\
Incidence of mucositis group II & $12(60 \%)$ & $17(86 \%)$ & 0.032 \\
Incidence of mucositis group III & $3(25 \%)$ & $11(55 \%)$ & 0.154 \\
Duration of parenteral nutrition (mean) & $16(0-32)$ & $26(13-40)$ & 0.002 \\
Duration of manifestation of mucositis (mean) & $6(0-19)$ & $12(0-30)$ & 0.003 \\
Grading of mucositis & 1.73 & 2.47 & 0.030 \\
\hline
\end{tabular}


Table 3 Mucositis temporal distribution in all patients

\begin{tabular}{lccc}
\hline Study group & Days of mucositis & Control group & Days of mucositis \\
\hline 1 & 6 & 1 & 12 \\
2 & 4 & 2 & 14 \\
3 & 5 & 3 & 15 \\
4 & 7 & 4 & 12 \\
5 & 8 & 5 & 10 \\
6 & 8 & 6 & 9 \\
7 & 7 & 7 & 12 \\
8 & 5 & 8 & 13 \\
9 & 4 & 9 & 14 \\
10 & 6 & 10 & 12 \\
11 & 9 & 11 & 11 \\
12 & 7 & 12 & 10 \\
13 & 6 & 13 & 12 \\
14 & 6 & 14 & 15 \\
15 & 4 & 15 & 9 \\
16 & 5 & 16 & 10 \\
17 & 4 & 17 & 14 \\
18 & 7 & 18 & 12 \\
19 & 8 & 19 & 14 \\
20 & 6 & 20 & 10 \\
\hline
\end{tabular}

Table 4 Secondary events to the administration of palifermin

\begin{tabular}{lcc}
\hline Side effects & $\begin{array}{c}\text { Study group } \\
\text { percentage (number) }\end{array}$ & $\begin{array}{c}\text { Control group } \\
\text { percentage }\end{array}$ \\
\hline Rash & $30 \%(6)$ & - \\
Erythema & $30 \%(6)$ & - \\
Altered taste & $10 \%(2)$ & - \\
Pain of the buccal and & $10 \%(2)$ & - \\
$\quad$ tongue mucosa & & \\
\hline
\end{tabular}

100-day survival rate was also very similar in both groups: one patient in the treatment group and two patients in the control group died before the 100th day after allergenic haematopoietic stem cell transplantation.

In summary, our data indicate that Palifermin may be most successful in reducing the incidence of mucositis in subjects categorized as group $2(P=0.032)$ and also in reducing the overall duration and severity of mucositis.

Observed adverse effects included skin rash, skin erythema and altered taste; two patients in the treatment group, in particular, experienced severe pain in the tongue, buccal mucosa and palate, measured as a value of 7 on the Visual Analogue Scale (Table 4).

\section{DISCUSSION}

This preliminary study seems to confirm the clinical efficacy of the Palifermin in the treatment of mucositis in paediatric patients. ${ }^{15,17}$

Parameters of comparison between the study group and the control group such as the mean duration of parenteral nutrition and the mean duration of mucositis were also reduced by administration of the drug. In addition, subjects receiving Palifermin also experienced less severe mucositis compared to the controls. Moreover, the incidence of mucositis in groups I, II and III was always lower in the treatment group compared to the control group. Although not statistically significant in groups 1 and 3, the trend observed in this study appears to indicate that Palifermin is likely to be beneficial in the reduction of mucositis overall (Figure 1). A larger study is needed verify this benefit. Mucositis is one of the most debilitating and expensive side effects

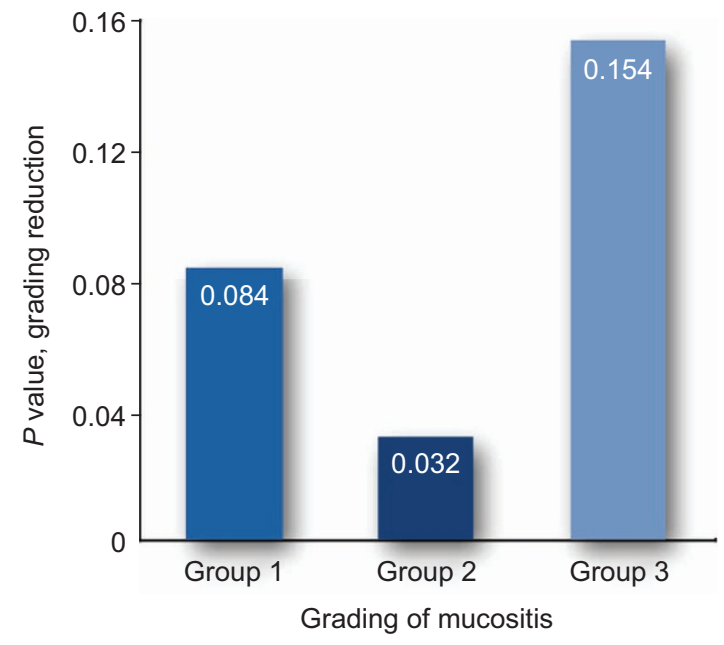

Figure $1 P$ value, incidence of grading reduction in the different groups.

to treat, and any reduction in its incidence, duration or severity is welcomed.

These observations could be directly related to the mechanism of action of palifermin, ${ }^{15,17}$ that, as we observed, operates at the level of the mucosa and not at the level of the pathogenetic mechanisms of the disease.

Moreover, the comparison between the treatment and control groups, shows that the drug drastically reduces the use of parenteral nutrition and the duration of the manifestations of mucositis itself. These findings allow us to conclude that palifermin, while not guaranteeing to eliminate mucositis, allows a remarkable improvement in the patient's condition, which is critical for individuals who are already highly debilitated by leukaemia and chemoradiotherapies. Moreover, the administration of palifermin could be considered generally safe and without significant complications.

In conclusion, palifermin cannot be recommended as a cure for mucositis (of any grade) with 'statistical certainty' due to the variability in the two groups.

We believe that through a multimodal approach of appropriate oral cavity care, i.e., with proper odontostomatological management ${ }^{31-32}$ application of prevention protocols, ${ }^{33}$ oral infection control ${ }^{34}$ and monitoring oral health, ${ }^{35}$ it is possible to improve the quality of life of children with leukaemia before, during and after systemic therapy.

1 McKenna SJ. Leukemia. Oral Surg Oral Med Oral Pathol Oral Radiol Endod 2000 89(2): 137-139.

2 Majhail NS, Rizzo JD, Lee SJ et al. Recommended screening and preventive practices for long-term survivors after hematopoietic cell transplantation. Bone Marrow Transplant 2012; 47(3): 337-341.

3 Bellm LA, Epstein JB, Rose-Ped A et al. Patient reports of complications of bone marrow transplantation. Support Care Cancer 2000; 8(1): 33-39.

4 Scully C, Sonis S, Diz PD. Oral mucositis. Oral Dis 2006; 12(3): 229-241.

5 Hernández-Fernández A, Oñate-Sánchez RE, Cabrerizo-Merino MC et al. Influence of oral health on mucositis in patients undergoing hematopoietic progenitor cell transplantation (HPCT). Med Oral Patol Oral Cir Bucal 2012; 17(1): 94-101.

6 Tanideh N, Tavakoli P, Saghiri MA et al. Healing acceleration in hamsters of oral mucositis induced by 5-fluorouracil with topical Calendula officinalis. Oral Surg Oral Med Oral Pathol Oral Radiol 2013; 115(3): 332-338.

7 Köstler WJ, Hejna M, Wenzel C et al. Oral mucositis complicating chemotherapy and/ or radiotherapy: options for prevention and treatment. CA Cancer J Clin 2001; 51(5): 290-315.

8 Bez C, Demarosi F, Sardella A et al. GM-CSF mouthrinses in the treatment of severe oral mucositis: a pilot study. Oral Surg Oral Med Oral Pathol Oral Radiol Endod 1999. 88(3): 311-315. 
9 Rubenstein EB, Peterson DE, Schubert $\mathrm{M}$ et al. Clinical practice guidelines for the prevention and treatment of cancer therapy-induced oral and gastrointestinal mucositis. Cancer 2004; 100(9 Suppl): 2026-2046.

10 Cheng KK. Children's acceptance and tolerance of chlorhexidine and benzydamine oral rinses in the treatment of chemotherapy-induced oropharyngeal mucositis. Eur $\mathrm{J}$ Oncol Nurs 2004; 8(4): 341-349.

11 Djuric M, Hillier-Kolarov V, Belic A et al. Mucositis prevention by improved dental care in acute leukemia patients. Support Care Cancer 2006; 14(2): 137-146.

12 Miller MM, Donald DV, Hagemann TM. Prevention and treatment of oral mucositis in children with cancer. J Pediatr Pharmacol Ther 2012; 17(4): 340-350.

13 Spielberger R, Stiff $\mathrm{P}$, Bensinger $\mathrm{W}$ et al. Palifermin for oral mucositis after intensive therapy for hematologic cancers. N Engl J Med 2004; 351(25): 2590-2598.

14 Meropol NJ, Somer RA, Gutheil J et al. Randomized phase I trial of recombinant human keratinocyte growth factor plus chemotherapy: potential role as mucosal protectant. J Clin Oncol 2003; 21(8): 1452-1458.

15 Schmidt E, Thoennissen NH, Rudat A et al. Use of palifermin for the prevention of high-dose methotrexate-induced oral mucositis. Ann Oncol 2008; 19(9): 1644-1649.

16 Blijlevens N, Sonis S. Palifermin (recombinant keratinocyte growth factor-1): a pleiotropic growth factor with multiple biological activities in preventing chemotherapy- and radiotherapy-induced mucositis. Ann Oncol 2007; 18(5): 817-826.

17 Langner S, Staber P, Schub N et al. Palifermin reduces incidence and severity of oral mucositis in allogeneic stem-cell transplant recipients. Bone Marrow Transplant 2008; 42(4): 275-279.

18 Miller MM, Donald DV, Hagemann TM. Prevention and treatment of oral mucositis in children with cancer. J Pediatr Pharmacol Ther 2012; 17(4): 340-350.

19 Srinivasan A, Kasow KA, Cross S et al. Phase I study of the tolerability and pharmacokinetics of palifermin in children undergoing allogeneic hematopoietic stem cell transplantation. Biol Blood Marrow Transplant 2012; 18(8): 1309-1314.

20 St. Jude Children's Research Hospital. Dose escalation study of palifermin in pediatric research participants undergoing allogeneic hematopoietic stem cell transplantation. Memphis: St. Jude Children's Research Hospital. Available at http://clinicaltrials.gov/ ct2/show/results/NCT00701688. (accessed 23 May 2013).

21 Blazar BR, Weisdorf DJ, DeFor T et al. Phase 1/2 randomized, placebo-control trial of palifermin to prevent graft-versus-hostdisease (GVHD) after allogeneic hematopoietic stem cell transplantation (HSCT). Blood 2006; 108(9): 3216-3222.

22 Vadhan-Raj S, Trent J, Patel S et al. Single dose palifermin prevents severe oral mucositis during multicycle chemotherapy in patients with cancer. Ann Intern Med 2010; 153(6): 358-367.

23 Spielberger R, Stiff $\mathrm{P}$, Bensinger $\mathrm{W}$ et al. Palifermin for oral mucositis after intensive therapy for hematologic cancers. N Engl J Med 2004; 351(25): 2590-2598.
24 Jayachandran S, Balaji N. Evaluating the effectiveness of topical application of natural honey and benzydamine hydrochloride in the management of radiation mucositis. Indian J Palliat Care 2012; 18(3): 190-195.

25 Roopashri G, Jayanthi K, Guruprasad R. Efficacy of benzydamine hydrochloride, chlorhexidine and povidone iodine in the treatment of oral mucositis among patients undergoing radiotherapy in head and neck malignancies: a drug trail. Contemp Clin Dent 2011; 2(1): 8-12.

26 Kazemian A, Kamian S, Aghili M et al. Benzydamine for prophylaxis of radiationinduced oral mucositis in head and neck cancers: a double-blind placebo-controlled randomized clinical trial. Eur J Cancer Care 2009; 18(2): 174-178.

27 Kin-Fong Cheng K, Ka Tsui Yuen J. A pilot study of chlorhexidine and benzydamine oral rinses for the prevention and treatment of irradiation mucositis in patients with head and neck cancer. Cancer Nurs 2006; 29(5): 423-430.

28 World Health Organization. Handbook for Reporting Results of Cancer Treatment. Geneva: World Health Organization, 1979.

29 McCrum-Gardner E. Which is the correct statistical test to use? Br J Oral Maxillofac Surg 2008; 46(1): 38-41.

30 Yamagata K, Onizawa K, Yanagawa T et al. A prospective study to evaluate a new dental management protocol before hematopoietic stem cell transplantation. Bone Marrow Transplant 2006; 38(3): 237-242.

31 Baldoni M, Lauritano D. [Odontostomatologic management of hemato-oncologic pediatric patients]. Minerva Pediatr 2007; 59(5): 445-446. Italian.

32 Lauritano D, Silvestre FJ, Borgia R et al. [Oral manifestation in neutropenic patients]. Dental Cadmos 2007; 75: 43-47. Italian.

33 Franceschini FG, Lauritano D, Panzeri $C$ et al. [Preventive dentistry coordination of Monza and Brianza]. Prev Assist Dent 2008; 34: 15-23. Italian.

34 Franceschini FG, Lauritano D, Baldoni M. [Bacterium and virus concentration in mouth of laeukemic pediatric patients]. Prev Assist Dent 2008; 34: 7-20. Italian.

35 Lauritano D, Petruzzi M. Decayed, missing and filled teeth index and dental anomalies in long-term survivors leukaemic children: a prospective controlled study. Med Oral Patol Oral Cir Bucal 2012; 17(6): e977-e980.

This work is licensed under a Creative Commons Attribution-NonCommercial-NoDerivative Works 3.0 Unported License. To view a copy of this license, visit http:// creativecommons.org/licenses/by-nc-nd/3.0 